[0212-7199 (2007) 24: 9; pp 428-430] ANALES DE MEDICINA INTERNA Copyright (C) 2007 ARAN EDICIONES, S.L.

AN. MED. InTERna (Madrid) Vol. 24, N. ${ }^{\circ}$ 9, pp. 428-430, 2007

\title{
Efectos adversos inducidos por gadopentotato dimeglumina en resonancia magnética
}

\author{
A. CAMPOS, F. DE LA GUARDIA ${ }^{1}$, J. REYES, C. FERRERO ${ }^{1}$ \\ Servicio de Alergia. ${ }^{l}$ Centro de Diagnóstico por la Imagen Eresa. Hospital La Fe. \\ Valencia
}

\section{RESUMEN}

Fundamento y objetivos: Se considera que las sales de gadolinio utilizadas como medio de contraste en los estudios de resonancia magnética (RM) presentan un índice de reacciones adversas inferior a los medios de contraste yodado. El objetivo principal de este estudio es determinar la frecuencia e intensidad de las reacciones adversas relacionadas con la administración de gadopentotato dimeglumina (Gd-DTPA) y compararla con la tasa de reacciones adversas inducidas por los medios de contaste yodado.

Material y métodos: Durante el periodo comprendido entre el 1 de enero de 2005 hasta el 30 de junio del 2006 se recogieron los efectos adversos de todos los estudios mediante RM en los que se aplicó contraste de Gd-DTPA, programados o urgentes, realizados en 11 Centros de Diagnostico por la Imagen Eresa situados en las provincias de Castellón y de Valencia. En dicho período se recogieron también los efectos adversos inducidos por las sales de yodo.

Resultados: Se realizaron un total de 54.909 estudios con resonancia magnética en los diferentes centros de estudio, y 12.791 estudios con MCI. El total de reacciones adversas observado fue de $62(0,11 \%)$ y 47 $(0,36 \%)$ respectivamente. El perfil de reacciones adversas con ambos medios de contraste fue similar, excepto en las reacciones moderadas donde objetivamos un mayor porcentaje de edema palpebral y disnea con la utilización de MCI que con Gd-DTPA. No se objetivó ningún caso de anafilaxia ni exitus.

Conclusiones: Los datos aportados por esta serie avalan un perfil de seguridad del Gadolinio superior al de los MCI.

PALABRAS CLAVE: Reacciones adversas. Alergia. Medios de contraste. Gadolinio. Yodo. Resonancia magnética.
ADVERSE REACTIONS INDUCED BY GADOPENTATATE DIMEGLUMINE IN MAGNETIC RESONANCE IMAGING

\section{ABSTRACT}

Background and objectives: It is considered that the Gadolinium salts used as a contrast medium in the Magnetic Resonance Imaging (MRI) studies present an index of adverse reactions below the iodine contrast mediums. The main objective of this study is to determine, in our medium, the frequency and intensity of the adverse reactions related to the administration of Gadopentotate Dimeglumine (Gd-DTPA) and compare it with the rate of adverse reactions induced by the iodine contrast mediums.

Material and methods: During the period comprised between 1st of January 2005 and 30th of June 2006, the adverse affects were collected for all the studies by means of MRI in which the Gd-DTPA contrast was applied, programmed or urgent, performed in Eresa Diagnosis Centres by Image located ( 11 centre in total) in the provinces of Castellón and Valencia. The adverse effects induced by the iodine salts were also collected in this period.

Results: A total of 54,909 studies with magnetic resonance were performed in the refered study centres, and 12,791 studies with MCI. The total of adverse reactions observed was $62(0.11 \%)$ and $47(0.36 \%)$ respectively. The profile of adverse reactions with both contrast mediums was similar, except in the moderate reactions where we objectively observed a greater percentage of palpebral edema and dyspnea with the usage of MCI than with Gd-DTPA. No cases of anaphylaxia or exitus were observed.

Conclusions: The data contributed by this series support a safety profile of Gadolinium superior to that of the MCI in line with the results shown by extensive published series.

KEY WORDS: Adverse reaction. Allergy. Contrast media. Gadolinium. Iodine. Magnetic resonance imaging.

Campos A, de la Guardia F, Reyes J, Ferrero C. Efectos adversos inducidos por gadopentotato dimeglumina en resonancia magnética. An Med Interna (Madrid) 2007; 24: 428-430.

\section{INTRODUCCIÓN}

Desde su introducción a finales de los años 80 , se considera que las sales de gadolinio $(\mathrm{Gd})$ utilizadas como medio de contraste en los estudios de resonancia magnética (RM) presentan un bajo índice de reacciones adversas, y que la frecuencia y gravedad de éstas son mucho menores que las pro- ducidas por los medios de contraste yodado (MCI). La incidencia global de efectos adversos en EE.UU. y Europa varían según las series publicadas y el lugar de estudio, pero se sitúan entre un $1,47 \%$ a $0,066 \%(1,2)$.

En nuestro país sólo disponemos de un estudio publicado en 1991 (3) acerca de la valoración de los efectos adversos a corto plazo del Gd-DTA en RM en 529 pacientes, de los cua- 
les exclusivamente uno refirió una discreta sensación nauseosa que no precisó de tratamiento.

El objetivo principal de este estudio es determinar la frecuencia e intensidad de las reacciones adversas relacionadas con la administración de Gd-DTPA durante la realización de RM en 11 Centros de Diagnostico por la Imagen ERESA (CDI-Eresa) situados en las provincias de Valencia y de Castellón. Así mismo, compararla con la tasa de reacciones adversas inducidas por MCI en el mismo periodo de tiempo en dichos centros. A efectos de comparabilidad los efectos adversos se clasificaron en subjetivas, leves, moderadas y severas.

\section{MATERIAL Y MÉTODOS}

Durante el periodo comprendido entre el 1 de enero de 2005 y el 30 de junio del 2006 se recogieron los efectos adversos de todos los estudios mediante RM en los que se aplicó contraste de Gd-DTPA (Gadopentotato de Dimeglumina: Magnevist ${ }^{\circledR}$. Laboratorio Schering. Berlín), programados o urgentes, realizados en los Centros de Diagnostico por la Imagen Eresa situados en las provincias de Castellón y de Valencia (4 y 7 centros respectivamente). En dicho período se recogieron también los efectos adversos inducidos por las sales de yodo.

El periodo de observación comprendía desde la administración del contraste y hasta 30 minutos posteriores. Los datos se recogieron en una ficha interna uniforme para todos los centros de fácil cumplimentacion. Con idéntico proceder se recogieron las reacciones adversas relacionadas con la administración de MCI en tales centros durante dicho periodo de tiempo.

La recogida de datos corrió a cargo del personal de enfermería encargado de su administración, supervisado por el médico especialista responsable de las exploraciones y del tratamiento si procedía. En todos los casos constaba la edad y los posibles antecedentes de reacciones previas con el uso de MCI, salvo en un pequeño número de casos que no se pudo recuperar. El Gd-DTPA se administró una dosis de 0,1 $\mathrm{mmol} / \mathrm{kg}$ de peso. El numero total de exploraciones realizadas se obtuvo consultando la base de datos de Eresa.

A efectos de comparabilidad los efectos adversos se clasificaron siguiendo la propuesta por Murphy en subjetivas, leves, modceradas y severas. (2)

\section{RESULTADOS}

Durante el período de estudio citado se sometieron a estudios con resonancia magnética un total de 54.904 pacientes en los diferentes centros de estudio, y 12.791 a MCI. El total de reacciones adversas observado fue de $62(0,11 \%)$ y 47 $(0,36 \%)$ respectivamente. En la tabla I se recogen los datos desglosados según el tipo de reacción: subjetiva, leve, moderada y severa. Las reacciones severas para Gd y MCI fue de 0,005 y $0,022 \%$ respectivamente. No hubo ningún caso de parada cardiorrespiratoria en ambos grupos.

\section{DISCUSIÓN}

Los datos obtenidos en nuestro estudio ponen de manifiesto una tasa de reacciones adversas con Gd-DTPA, tres veces
TABLA I

TIPO Y FRECUENCIA DE REACCIONES ADVERSAS SEGÚN SU GRAVEDAD

\begin{tabular}{llcc}
\hline Tipo & Signos y síntomas & Gadolinio (\%) & MCI (\%) \\
\hline Subjetiva & Intranquilidad & 8,65 & 1,52 \\
& Sensación de desmayo & 2,46 & 1,52 \\
& Rigidez & 0 & 1,52 \\
& Naúseas/vómitos & 21,02 & 20,06 \\
& Cefalea & 2,46 & 1,52 \\
& Dolor abdominal & 1,23 & 3,04 \\
& Dolor torácico & 0 & 1,52 \\
Leve & Tos & 2,46 & 1,52 \\
& Prurito faríngeo/odinofagia & 4,93 & 0 \\
& Palidez/sudoración & 6,18 & 4,58 \\
& Rubefacción & 6,18 & 0 \\
& Urticaria/rash cutáneo & 38,27 & 49,34 \\
Moderado & Edema palpebral & 1,23 & 3,08 \\
& Disnea & 0 & 4,63 \\
Severo & Edema de glotis/facial & 4,93 & 4,63 \\
& Edema cerebral & 0 & 1,52 \\
& Broncoespasmo & 0 & 0 \\
& Shock & 0 & 0 \\
& Parada cardiorespiratoria & 0 & 0 \\
\hline
\end{tabular}

inferior a las inducidas por los MCI, con una diferencia estadísticamente significativa $(\mathrm{p}<0,05)$ en consonancia con los datos publicados en la literatura.

Niendorf y cols. (1) de entre 13.439 pacientes, objetivaron 239 reacciones adversas asociadas con la administración de Gd-DTPA i.v. a dosis de 0,1 a $0,2 \mathrm{mmol} / \mathrm{kg}$, lo que representa $1,47 \%$ de los pacientes.

Murphy y cols. (2) encontraron una tasa de 0,066\% de reacciones adversas en 687.255 administraciones. Sin embargo, Chu WC y cols. (4) en una serie de 2.049 administraciones, presentaron un $15 \%$ de reacciones adversas.

En nuestro tranajo, el perfil de reacciones adversas con ambos medios de contraste fue similar, excepto en las reacciones moderadas donde objetivamos un mayor porcentaje de edema palpebral y disnea con la utilización de MCI que con Gd-DTPA $(p<0,05)$.

Aunque no hubo ningún caso de shock, broncoespasmo ni parada cardiorrespiratoria en nuestro estudio, debemos de tener presente dicha posibilidad, ya que existen publicados casos anecdóticos de reacciones adversas con compromiso vital (5-9) e incluso exitus (10).

Debido al menor riesgo de aparición de efectos adversos con Gd-DTPA que con los MCI, algunos autores (11-13), preconizan el uso de contrastes paramagnéticos en pacientes con antecedentes de reacción adversa a MCI.

Por otra parte Schiavino y cols. (14) presentan un caso de reacción adversa tras la administración de Gd-DTPA detectándose la positividad de las pruebas cutáneas intradérmicas y el test de transferencia pasiva de Prausnitz-Kustner, lo cual le permite a los autores aventurar la hipótesis de una implicación inmunológica IgE mediada en este caso. En el momento actual, no disponemos de datos que avalen este mecanismo inmunológico y por tanto, no parece razonable plantear el uso extendido de pruebas cutáneas en todos los casos. 
Tampoco existe un consenso acerca del manejo del paciente con antecedentes de reacciones previas con sales de Gd. La Academia Americana de Neuroradiología (2) recoge diversas posturas entre sus asociados ante una reacción adversa previa por $\mathrm{Gd}$ : un $9,5 \%$ prohíbe de forma absoluta la utilización futura de este contraste, un $36 \%$ no realiza ninguna medida de precaución, un $5,6 \%$ propone una pauta de premedicación con antihistamínicos $\mathrm{H} 1$, y un $49 \%$ premedican al paciente con glucocorticoides y antihistamínicos $\mathrm{H} 1$ según diferentes pautas y dosis. Niendorf y cols. (1) observaron que en pacientes con antecedentes de reacción adversa con sales de yodo la incidencia de presentar una reacción adversa con sales de Gd aumentaba a 2,6\%.

\section{Bibliografía}

1. Niendorf HP, Alazán A, Balzer T, Geens V. Safety and risk of gadolinium-DTPA: extended clinical experience after more than 20 million applications. Magn Reson Med 1991; 22: 222-8.

2. Murphy KP, Szopinski KT, Cohan RH, Mermillod B,Ellis JH. Occurrence of adverse reactions to gadolinium-based contrast material ad management of patients at increased risk: A survey of the American Society of Neuroradiology Fellowship Directors. Acad Radiol 1999; 1: 656-4.

3. Dualde D, Martí L, Poyatos C, Ronchera C, Morote V, Caballero E, et al. Valoración de los efectos adversos a corto plazo del gadolinio-DTPA en RM. Radiología 1991; 33: 653-5.

4. Chu WC, Lam WW, Metreweli C. Incidence of adverse events after i.v. injection of MR contrast agents in a Chinese population A comparision between gadopentetate and gadodiamide. Acta Radiol 2000; 41: 662-6.

5. Campos A, Aznar L, Alamar R, Castelló JV. Reacción anafilactoide tras gadopentetato dimeglumina i.v. durante Resonancia Magnética. Med Clin (Barc) 1996; 106: 197.

6. Wiss KL. Severe anaphylactoid reactions after iv Gd-DTPA. Mag Reson Imaging 1990; 8: 817-8.

7. Thisler S, Hoffman JC. Anaphylactoid reactions to IV Gadopentetate Dimeglumine. AJNR 1990; 11: 1167-8.

8. Tardy B, Guy C, Barral G. Anaphylactic shock induced by intravenous Gadopentetate Dimeglumine. Lancet 1992; 339: 494.

9. Omohundro JE, Elderbrook MK, Ringer TV. Laryngospasm after administration of gadopentetate dimeglumine. J Magn Reson Ima-
De manera empírica, ante pacientes con reacciones adversas previas a gadolinio, nosotros planteamos la realización de la pauta de premedicación preconizada por Greenberger (15) y validada para el caso de los MCI ante pacientes con antecedentes de reacciones adversas de tipo anafilactoide.

Es de esperar la introducción en nuestro país de otras sales de Gd, especialmente las de tipo no iónico cuyo perfil de seguridad es superponible al de Gd-DTPA, (16).

En conclusión, los datos aportados por nuestro trabajo avalan un perfil de seguridad del gadolinio superior al de los MCI.

ging 1992; 2: 729-30.

10. Jordan RM, Mintz RD. Fatal reaction to gadopentetate dimeglumine. AJR 1996; 26: 806-10.

11. Sasamura H, Hashimoto S, Kuribayashi S, Ueno K, Takase A, Hayashi $\mathrm{M}$, et al. Use of Gadolinium Contrast Adrenal Venography for the Assesment of Primary Aldosteronism in a Patient with Iodine Allergy. Endocrine Journal 2004; 51: 487-92.

12. Arat A, Cekirge HS, Saatci I. Gadodiamide as an alternative contrast medium in cerecral angiography in a patient with sensitivity to iodinated contrast medium. Neuroradiol 2000; 42: 34-7.

13. Spinosa DJ, Kaufmann JA, Hartwell GD. Gadolinium chelates in angiography and International Radiology: A useful alternative to iodinated contrast media for angiography. Radiol 2002; 223: 319-25.

14. Schiavino D, Murzilli F, Del Ninno M, Buonomo A, Roncallo C, Pollastrini E, et al. Demonstration of an IgE-mediated immunological pathogenesis of a severe adverse reaction to gadopentetate dimglumine. J Investig Allergol Clin Immunol 2003; 13: 292.

15. Greenberger P, Paterson R. The prevention of immediate generalized reactions to radiocontrast in high risk patients. J Allergy Clin Immunol 1992; 87: 867-72.

16. Niendorf HP, Alazán A, Balzer T, Claus W, Cornelius I. Safety and risk of gadolinium-DTPA: extended clinical experience after more than 5,000,000 aplications. In Felix R, Heshiki A, Hosten H, Hricak H (eds). Magnevist Monograph 1994. London: Blackwell Science. p. 21-30. 